\title{
Coherent Structures in Interacting Vortex Rings
}

\author{
Jian Deng, ${ }^{1, *}$ Jingyu Xue, ${ }^{1}$ Xuerui Mao ${ }^{2}$ and C. P. Caulfield ${ }^{3,4}$ \\ ${ }^{1}$ Department of Mechanics, Zhejiang University, \\ Hangzhou 310027, People's Republic of China \\ ${ }^{2}$ Department of Mechanical, Materials and Manufacturing Engineering, \\ the University of Nottingham, University Park, Nottingham NG7 2RD, UK \\ ${ }^{3} B P$ Institute, University of Cambridge, \\ Madingley Road, Cambridge CB3 OEZ, UK \\ ${ }^{4}$ Department of Applied Mathematics 83 Theoretical Physics, \\ University of Cambridge, Centre for Mathematical Sciences, \\ Wilberforce Road, Cambridge CB3 0WA, UK
}

(Dated: February 9, 2017)

We investigate experimentally the nonlinear structures which develop from interacting vortex rings induced by a sinusoidally oscillating ellipsoidal disk in fluid at rest. We vary the scaled amplitude or Keulegan-Carpenter number, $0.3<K C=$ $2 \pi A / c<1.5$, where $A$ is the oscillation amplitude and $c$ is the major diameter of the disk, and the scaled frequency or Stokes number, $100<\beta=f c^{2} / \nu<1200$, where $f$ is the frequency of oscillation and $\nu$ is the kinematic viscosity. Broadly consistent with global linear stability analyses, highly organized nonlinear structures with clear azimuthal wavenumber emerge as sequential vortex rings are shed from the disk. These organized structures exhibit wavenumbers ranging from $m=2$ to $m=9$, and can be further divided into two distinct classes, distinguished by the phase and symmetry properties above and below the disk. We find some discrepancies between experiments and linear stability analysis, due to the inherent nonlinear mechanisms in the experiments, particulary on the boundary between the two branches, presenting unevenly distributed flow structures along the azimuthal direction.

\footnotetext{
* Author to whom correspondence should be addressed. Electronic address: zjudengjian@zju.edu.cn
} 


\section{INTRODUCTION}

Vortex rings are canonical structures in fluid mechanics, due to their broad appearance in the natural world, ranging from volcanic eruptions [1] to the propulsion of jellyfish and other aquatic creatures [2-5]. Vortex rings also have wide engineering applications, for example in underwater drilling, combustion, artificial olfaction and combustion [6]. The dynamics of a vortex ring has been found to be dominated by large-scale structures, for instance in the breakdown of a vortex ring [7], and in turbulent transport and subsequently the efficient and environmentally-clean operation of a combustor [8]. The manipulation, either excitation or suppression, of such large-scale coherent structures by means of passive or active control has been found to enhance mass entrainment rate [9-12] or reduce noise [13]. In Ref. [9], Grinstein et al. used a set of five pairs of split delta-wings installed at the jet exit to stimulate the preferred large coherent structures which have a five-fold symmetry in the azimuthal direction. In Ref. [11], Önder \& Meyers applied three actuators near the nozzle exit and observed a hexagram deformation of the vortex ring, as well as finer secondary structures further downstream.

Although there is still a lack of understanding of the underlying growth mechanisms for such azimuthally periodic structures, it is reasonable to suppose that the observed, inherently nonlinear structures, not least because of their observed symmetries, can be considered to be the consequence of linear instabilities triggered by infinitesimally small perturbations. Although at sufficiently small Reynolds numbers, vortex rings are generally considered to be highly robust[14], it is also well-known that at sufficiently high Reynolds number, vortex rings are unstable, and indeed can become fully turbulent [15]. A vortex ring propagating through an otherwise quiescent fluid was shown to be prone to the socalled Moore-Saffman-Tsai-Widnall (MSTW) instability [6, 15-18], which was originally identified through consideration of a straight vortex tube subject to a straining field in a plane perpendicular to the tube axis[17]. The underlying instability dynamics can be much more complicated when vortex rings interact either with flow boundaries or with each other, with multiple structures with non-zero azimuthal wavenumber being observed both experimentally $[19,20]$ and numerically [21-23]. Indeed, strong interactions between vortex rings, such as reconnection, leading to a change in their topology, have been found to affect key properties, such as intermittency and scalar mixing, of the ensuing turbulence[24].

In an isolated vortex ring, the azimuthal wavenumber observed in experiments can be as large as $m=12$ [25], while the highest azimuthal wavenumber observed in the head-on collision of two vortex rings is $m=20$ [20]. However, to the best of our knowledge, no previous publication has reported a wavenumber less than 6 , which appears to be a cut-off value for the isolated vortex ring instabilities.

Recently, we proposed a new model to investigate the instabilities arising from interacting vortex rings, generated by a periodically oscillating oblate spheroid disk [26]. At sufficiently low oscillation frequencies, the vortex ring loses axisymmetry due to a single branch of instability, while at higher oscillation frequencies, and hence stronger ring-ring interaction, the onset of non-trivial azimuthal structure is associated with multiple qualitatively distinct instabilities, with azimuthall wavenumbers ranging from $m=2$ to $m=10$. The associated nonlinear coherent structures were investigated by three-dimensional direct simulations, and in Fig.1, we show two typical cases corresponding to two qualitatively different branch- 

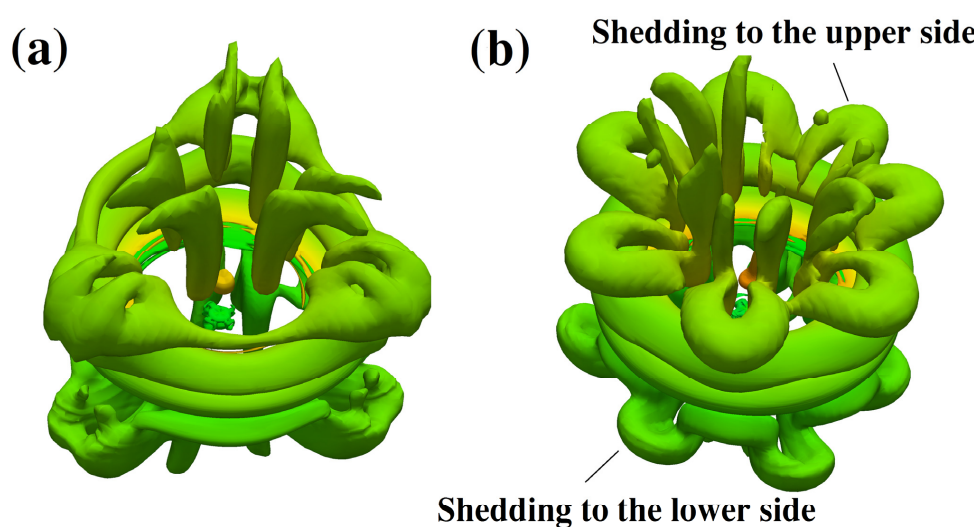

FIG. 1. (Color online) Vortical structures obtained by three-dimensional simulations for (a) $(K C, \beta)=(0.735,500)$ and $(\mathrm{b})(K C, \beta)=(0.879,500)[26]$. Note that the vortical structures shed above and below the disk are in phase or symmetric in (a) and out of phase or asymmetric in (b). $K C$ and $\beta$ are defined in (1).

es within the multiple instability regime (see [26] for further details). Here the symmetric (in-phase) (in Fig.1a) and asymmetric (out-of-phase) (in Fig.1b) structures on either side of the disk can be clearly identified. Here we develop an experimental model to visualize thee vortex rings in this high frequency regime, as we believe these high azimuthal wavenumber instabilities are both inherently interesting and highly relevant in various applications associated with vortex ring flows.

\section{PROBLEM FORMULATION AND METHODOLOGY}

Following the setup in [26], we fix the aspect ratio of the oblate spheroid at $A R=b / c=$ 0.1 , where $b$ and $c$ are the minor and major diameters of the oblate spheroid, respectively. The spheroid oscillates vertically with a frequency $f$ and an amplitude $A$, periodically shedding vortex rings from either side. This flow is characterized by two independent parameters: $\beta$ the non-dimensional frequency or Stokes number; and $K C$, the non-dimensional amplitude or Keulegan-Carpenter number respectively, defined as

$$
\beta=\frac{f c^{2}}{\nu}, \quad K C=\frac{2 \pi A}{c},
$$

where $\nu$ is the kinematic viscosity of the fluid.

When there is no disturbance, the flow converges to an axisymmetric and time-periodic base state, whose stability can be examined using Floquet analysis. Specifically, any perturbation of this base flow can be decomposed as the sum of modes

$$
\mathbf{u}^{\prime}(r, \theta, z, t)=\sum_{m=0}^{+\infty} \mathbf{u}^{(m)}(r, z, t) e^{i m \theta}
$$

where $i=\sqrt{-1}$. Since the linear dynamics of modes with different $m$ are decoupled, Floquet analysis can be carried out independently at each prescribed azimuthal wavenumber. 


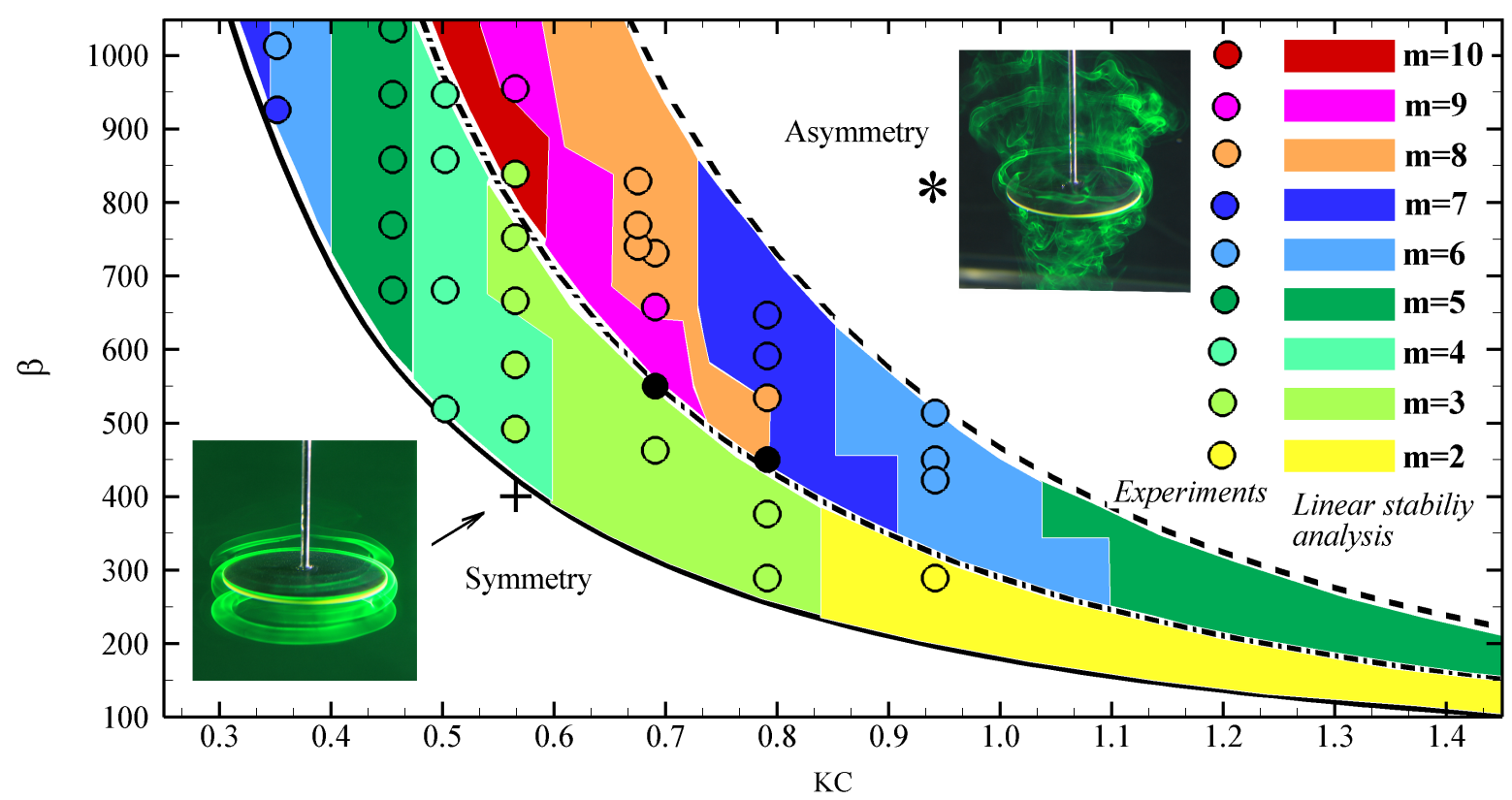

FIG. 2. (Color online) Regime diagram for axisymmetry-breaking instabilties in the $\beta-K C$ plane. The dashed line, $R e_{H}=\beta \cdot K C^{2}=467$ bounds, to the lower-left, the regime with $m=1$ instability. The solid line, $R e_{L}=\beta \cdot K C^{3 / 2}=180$ bounds, to the upper right, the symmetry regime $(m=0)$. Between these two lines, the filled circles with different colors mark different wavenumbers visualized by experiments, and the blocks with different colors represent the regimes with different wavenumbers determined by linear stability analyses. The region between the two lines can be further divided into an 'in-phase' branch and an 'out-of-phase' branch by the dash-dotted line. The insets show the experimental visualizations of a typical symmetric flow at $(K C, \beta)=(0.565,405)$ $($ marked by +$)$, and an asymmetric flow at $(K C, \beta)=(0.942,816)$ (marked by $*)$.

To determine the structure of the most unstable mode and its growth rate, we define a $(T$-periodic) operator $\mathbf{A}$. This operator evolves a perturbation from $t=0$ to $t=T=1 / f$ by integrating forward in time the Navier-Stokes equations, linearized about the calculated axisymmetric base flow, and so formally:

$$
\mathbf{u}^{\prime}(T)=\mathbf{A}(T) \mathbf{u}^{\prime}(0) .
$$

The actual (real) growth rate of the most unstable mode can then be obtained as $\operatorname{Real}\left(\sigma_{\max }\right)=$ $T^{-1} \ln \left(\operatorname{Real}\left(\lambda_{\max }\right)\right)$, where $\lambda_{\max }$ is the leading (i.e. with largest real part) eigenvalue of $\mathbf{A}$. This dominant eigenvalue can be calculated by applying an Arnoldi method to a Krylov subspace constructed from 'snapshot' flow solutions of the linearised Navier-Stokes equations. Instability occurs when the Floquet multiplier $\mu_{m}=\exp \left(\sigma_{m} T\right)$ leaves the unit circle, i.e. $\left|\mu_{m}\right|>1$.

In the calculation of both the base flow and the Floquet instabilities, a spectral-element code is adopted to discretize the governing equations [27]. This code has been successfully applied to various flow problems [28-30]. The grid has 812 spectral elements, each of which is further decomposed into $64(8 \times 8)$ nodes, producing convergence of the growth rate with an accuracy of $0.5 \%$. In an effort to eliminate boundary effects, the far-field boundary is 
located $25 c$ from the disk centre.

Experiments are performed using a water tank with internal dimensions $60 \mathrm{~cm} \times 60 \mathrm{~cm} \times$ $70 \mathrm{~cm}$. The oblate spheroid 'disk', with $c=10 \mathrm{~cm}$ and $b=1 \mathrm{~cm}$ is attached at its center to a vertical stainless steel tube with a diameter of $0.5 \mathrm{~cm}$ at its lower end. The upper end of the stainless tube is attached to a slider, which is oscillated along a rail by means of a scotch yoke mechanism. For flow visualization, fluorescent particles are injected from a very thin slot $(0.5 \mathrm{~mm}$ width) along the edge of the disk. The particles are illuminated and the flow pattern is photographed in both top-view and side-view.

\section{FLOQUET STABILITY RESULTS}

As shown in Fig.2, the flow is stable and symmetric at sufficiently low $K C$ or $\beta$. This stable sub-space is bounded to the upper-right on the $K C-\beta$ plane by a solid line, which may be interpreted as a critical low- $\beta$ Reynolds number defined as: $R e_{L}=\beta \cdot K C^{3 / 2}=$ $(2 \pi)^{3 / 2} f A \sqrt{c A} / \nu=180$, where the characteristic length scale involves both $c$ and $A$. At high $K C$ or $\beta$, in the regime bounded on the lower-left by a dashed line, the flow is predicted to become asymmetric with the most unstable mode having $m=1$. This dashed line is defined by a different critical Reynolds number as $R e_{H}=\beta \cdot K C^{2}=(2 \pi)^{2} f A^{2} / \nu=467$, where since the oscillations are at high amplitude, the characteristic length scale is given by this amplitude $A$ alone. The region between these two curves can be further separated into two parts by a dash-dotted line. On both sides of this line, for a fixed value of $\beta$, as $K C$ is increased, the value of $m$ associated with the most unstable mode decreases. However, this decrease exhibits a jump at this line, clearly associated with a change of the dominant predicted instability branch.

\section{EXPERIMENTAL OBSERVATIONS}

The instability regime diagram discussed in section III is also supported by experimental observations, as shown by the insets of Fig.2 which are obtained from experiments at the corresponding parameters, although it is important to remember that the stability analysis is linear, while the structures observed in the experiments are inherently nonlinear. In the regime predicted to be axisymmetric, axisymmetric vortex rings are indeed observed to be shed from the disk. The fluorescent tracers following the symmetric vortex rings actually stay at a fixed location after shedding from the disk (as shown at the lower-left of Fig.2). Conversely, within the regime predicted by the linear stability analysis to be asymmetric with a primary $m=1$ instability, turbulent breakdown is actually observed in experiments (shown at the upper-right of Fig.2), suggesting the onset of an array of higher order instabilities developing on the theoretically predicted primary instability.

Perhaps the most interesting feature of the experimentally observed unstable flow is the intermediate-time appearance of large coherent structures which in turn break down to turbulence. In Fig.2, the most unstable azimuthal wavenumber predicted by linear theory is marked by different colours. For comparison, the experimentally observed wavenumbers of large coherent structures are marked with circles filled with different colours. There are clearly some discrepancies between the theory and experimental observations. For $K C=0.565$, 


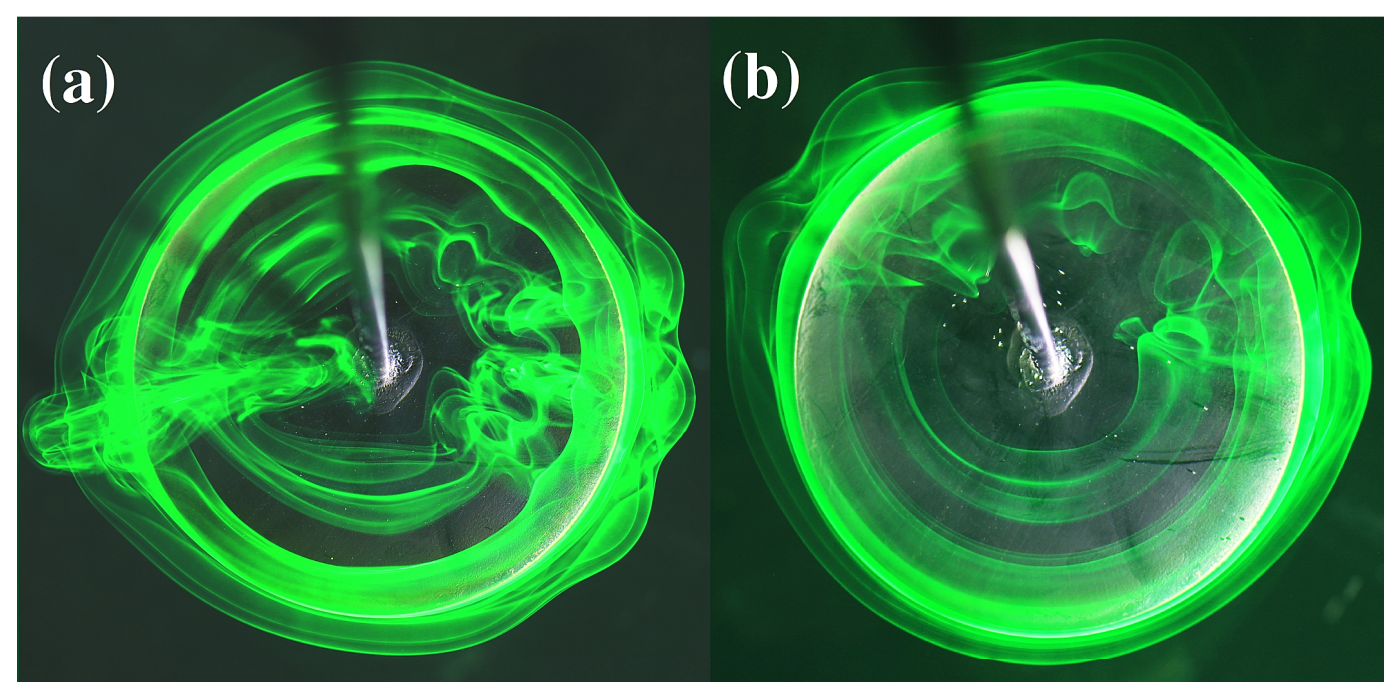

FIG. 3. Top-views of the experimental observation of unevenly azimuthally distributed multimode structures in unstable vortex rings, corresponding to the black filled circles in Fig.2: (a) $(K C, \beta)=(0.691,550),(\mathrm{b})(K C, \beta)=(0.791,450)$.

the linearly predicted wavenumber decreases from $m=4$ to $m=3$ for $\beta>650$, while experimentally, $m=3$ wavenumber structures are observed to persist until $\beta \simeq 850$. One natural possible explanation of this discrepancy is that nonlinearity plays a central role, a possibility further reinforced by the disordered flow patterns observed for parameters lying near the dash-dotted line in Fig.2. In this region of parameter space, the two qualitatively different 'in-phase' symmetric and 'out-of-phase' asymmetric instability branches with different wavenumber compete, leading to complex structures, as shown in Fig.3. These structures have non-trivial variation in their amplitudes azimuthally, and are clearly inherently nonlinear.

The finite amplitude structures for instabilities with flow parameters either side of the dash-dotted line in Fig.2 are qualitatively different. In Fig.4 we show experimental snapshots from the top view of the oscillating disk for flows with parameters falling between the solid and dash-dotted lines in Fig.2. Large coherent structures with $m=7,6,5,4,3$, and 2 are observed, the fluorescent tracers roll up and flow radially in clearly identifiable 'spokes' towards the center of the disk. These spokes are in phase on either side of the disk, and so we refer to this parameter region as the in phase branch. However, due to the in-phase nature of the fingers, it is not possible from top view to observe the vortical structures shedding from the lower side, although it is apparent, particularly in Fig.4(a) and (b), that there is a layering in the spokes, marking each of the individual oscillation cycles. Ideally, these fingers should be evenly spaced circumferentially by an angle of $2 \pi / m$, but we actually observe uneven distributions, most probably due to uncontrolled experimental perturbations or higher order effects, such as the secondary spoke in the top right corner of Fig.4(b).

For flows with parameters between the dashed and dash-dotted lines in Fig.2, snapshots are shown in Fig.5, taken at the lowest point of the oscillation. Coherent structures with wavenumber $m=9,8,7$, and 6 are observed. The vortical structures distorted from the vortex rings and shed to the upper side are clearly observed above the disk, while the vortex 


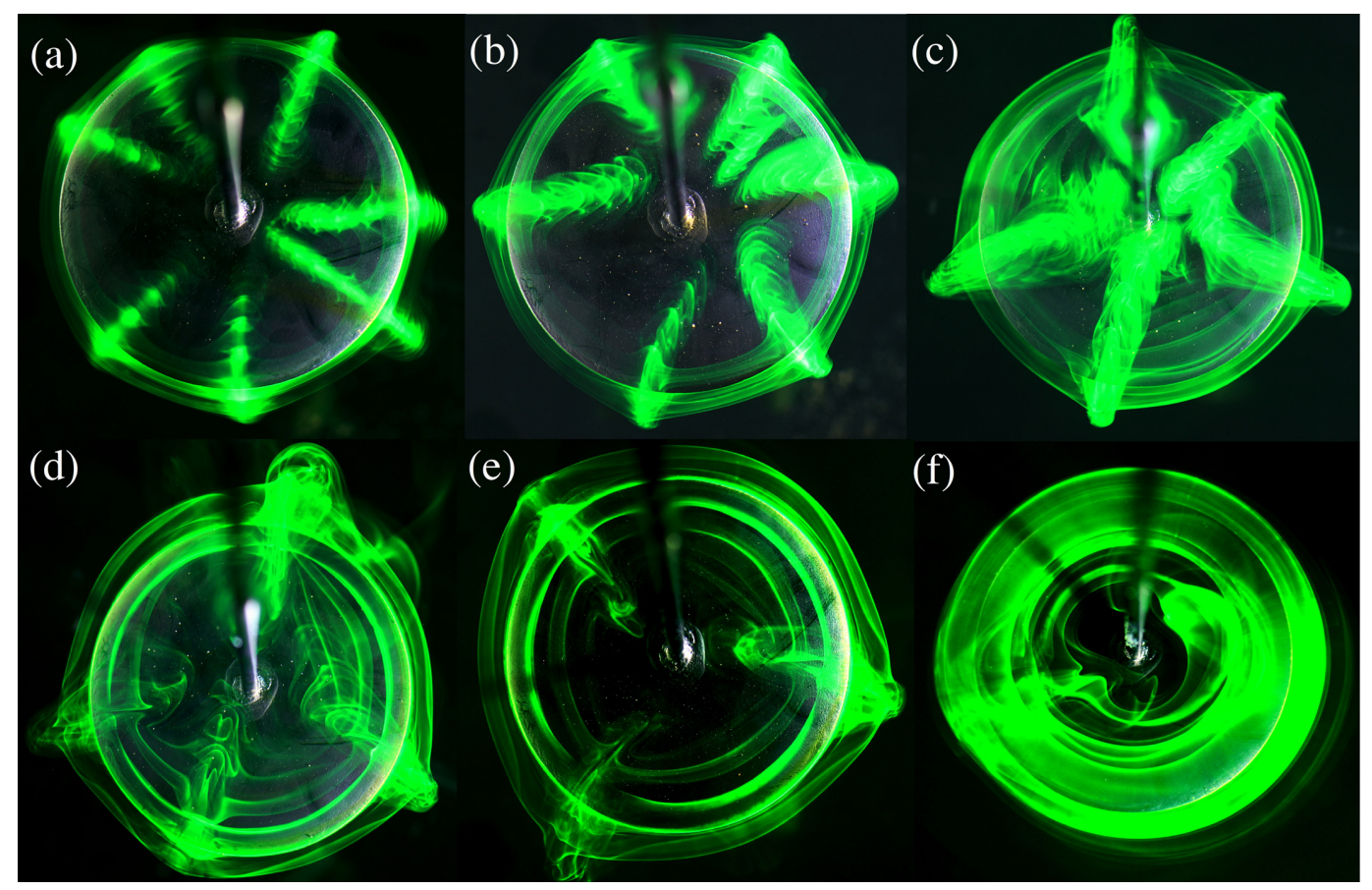

FIG. 4. Top-views of structures in the 'in-phase' branch (between solid and dash-dotted lines in Fig.2) with $(K C, \beta)=(0.352,926),(0.352,1013),(0.455,947),(0.502,947),(0.565,752)$ and $(0.942,289)$ for $(\mathrm{a}),(\mathrm{b}),(\mathrm{c}),(\mathrm{d}),(\mathrm{e})$ and $(\mathrm{f})$, respectively.

rings about to be shed to the lower side are earlier in their development, exhibiting wavy patterns along the edge of the disk. Crucially, these structures are out of phase above and below the disk, and so we refer to this branch of instability as the 'out-of-phase' branch. There is qualitative difference in the finite amplitude structures associated with the out-ofphase and in-phase branches, even for instabilities with the same azimuthal wavenumber, as is shown for $m=7$ in Fig.5(c) and Fig.4(a) and $m=6$ in Fig.5(d) and Fig.4(b).

The in-phase and out-of-phase branches can be even more clearly distinguished from side views of the flow structures. Fig. 6 shows the experimental side views of two typical cases corresponding to Fig.4(c) and Fig.5(c). It is also worth noting that in Fig.6(a), the flow structures again exhibit layering due to contributions from each oscillation, although this layering is not apparent at higher Reynolds number as shown in Fig.6(b). Finally, we note that the wavenumbers in experimental observations also agree well with previous three-dimensional direct numerical simulations [26].

\section{CONCLUSIONS}

We have experimentally visualized nonlinear coherent structures arising from interacting vortex rings shed from an ellipsoidal disk. These coherent structures exhibit broken axisymmetry with respect to the disk axis. As the scale of the rings increases, or equivalently as the $K C$ number increases, two qualitatively different branches of structures exhibiting a wide range of azimuthal wavenumber $m=2 \sim 9$ are identified, followed by an $m=1$ mode. 


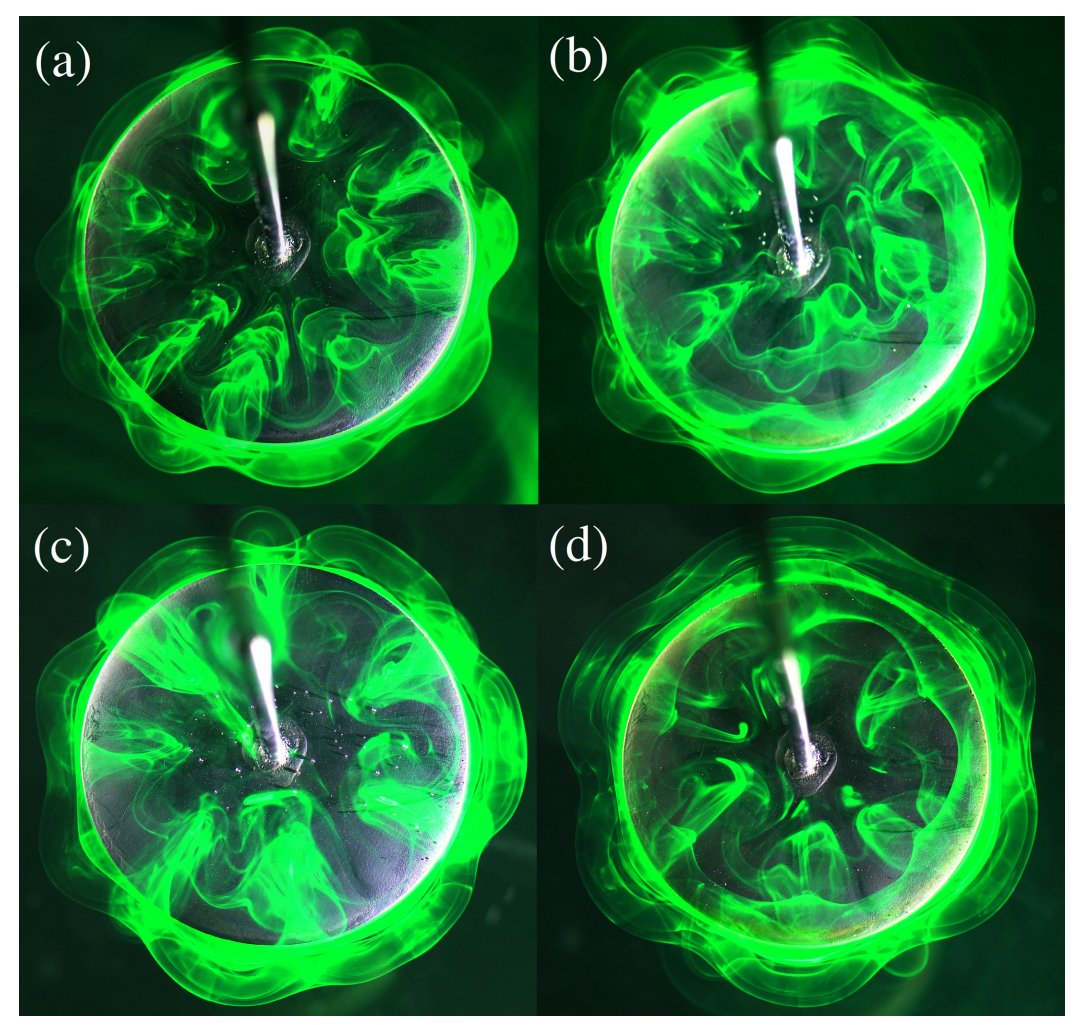

FIG. 5. Top-views of structures in the 'out-of-phase' branch as shown in Fig.2, with (a) $(K C, \beta)=$ $(0.691,658),(0.791,534),(0.791,591)$ and $(0.942,422)$ for $(\mathrm{a}),(\mathrm{b}),(\mathrm{c})$ and $(\mathrm{d})$, respectively.

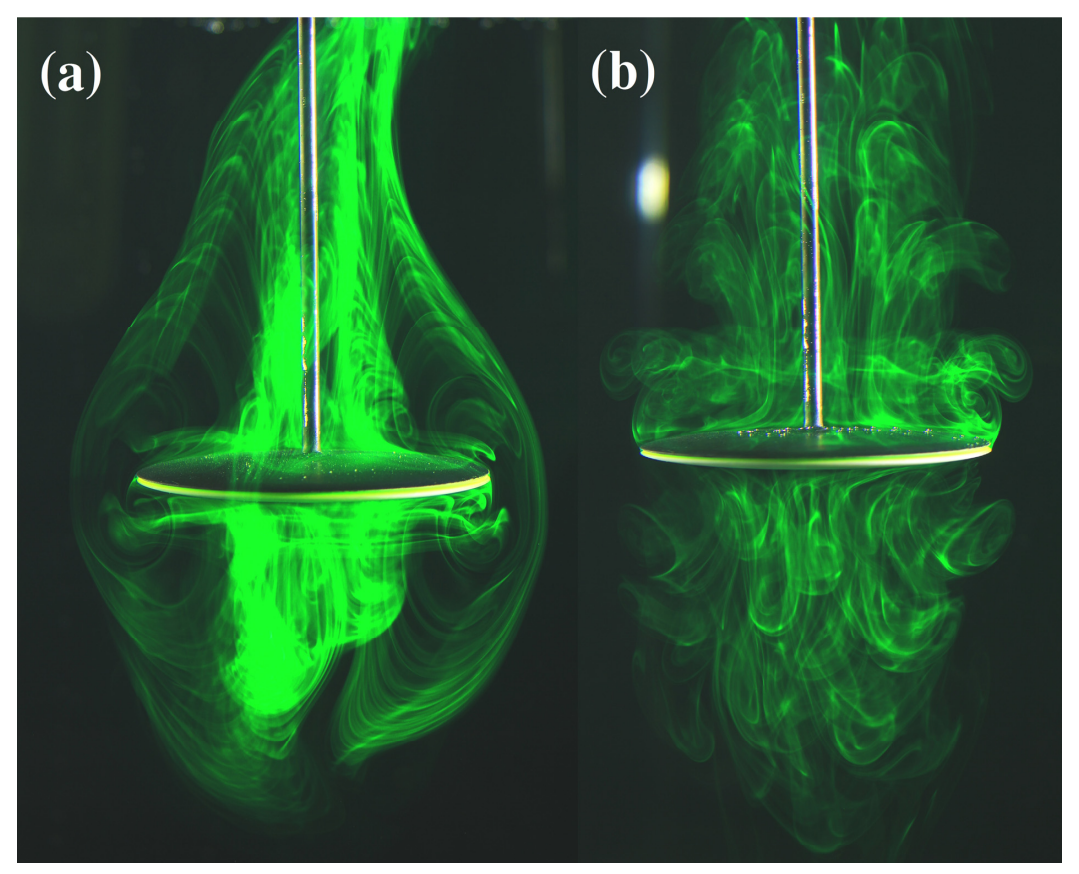

FIG. 6. Side-views of the flow structures for (a) $(K C, \beta)=(0.455,947)$ and (b) $(K C, \beta)=$ $(0.791,591)$. Equivalent top-view images are shown in Fig.4(c) and Fig.5(c) respectively. 
These two branches are characterised at finite amplitude by in-phase and out-of-phase distributions of structures above and below the oscillating disk, and in turn illustrate a further symmetry breaking, this time with respect to the disk plane. We have demonstrated conclusively that these two branches of instability can both grow in a real experimental flow, and also saturate at finite amplitude exhibiting non-trivial azimuthally periodic structure. Probing the secondary stability properties and pathways to transition of these finite amplitude structures is a key future avenue of research, particularly at higher Reynolds number.

\section{ACKNOWLEDGMENTS}

This research is supported by the Public Projects of Zhejiang Province (Grant No: 2015C31126) to conduct this research. Header data required to start the simulations shown in Fig.1, and data required to construct Fig.2, are available at https://doi.org/10.17863/CAM.7652.

[1] Frank I Perret, "Volcanic vortex rings," Sci. Am. 75, 4-5 (1913).

[2] PF Linden and JS Turner, "The formation of optimalvortex rings, and the efficiency of propulsion devices," Journal of Fluid Mechanics 427, 61-72 (2001).

[3] PF Linden and JS Turner, "optimalvortex rings and aquatic propulsion mechanisms," Proceedings of the Royal Society of London B: Biological Sciences 271, 647-653 (2004).

[4] Paul S Krueger, Ali A Moslemi, J Tyler Nichols, Ian K Bartol, and William J Stewart, "Vortex rings in bio-inspired and biological jet propulsion," in Advances in Science and Technology, Vol. 58 (Trans Tech Publ, 2008) pp. 237-246.

[5] Lydia A Ruiz, Robert W Whittlesey, and John O Dabiri, "Vortex-enhanced propulsion," Journal of Fluid Mechanics 668, 5-32 (2011).

[6] Karim Shariff and Anthony Leonard, "Vortex rings," Annu. Rev. Fluid Mech. 24, 235-279 (1992).

[7] P. Archer, T. Thomas, and G. Coleman, "Direct numerical simulation of vortex ring evolution from the laminar to the early turbulent regime," J. Fluid Mech. 598, 201 - 226 (2008).

[8] S Cj Crow and FH Champagne, "Orderly structure in jet turbulence," Journal of Fluid Mechanics 48, 547-591 (1971).

[9] FF Grinstein, EJ Gutmark, TP Parr, DM Hanson-Parr, and U Obeysekare, "Streamwise and spanwise vortex interaction in an axisymmetric jet. a computational and experimental study," Physics of Fluids (1994-present) 8, 1515-1524 (1996).

[10] Guillaume Balarac, Olivier Métais, and Marcel Lesieur, "Mixing enhancement in coaxial jets through inflow forcing: A numerical study," Physics of Fluids (1994-present) 19, 075102 (2007).

[11] Asim Önder and Johan Meyers, "Modification of vortex dynamics and transport properties of transitional axisymmetric jets using zero-net-mass-flux actuation," Physics of Fluids (1994present) 26, 075103 (2014).

[12] Asim Önder and Johan Meyers, "Optimal control of a transitional jet using a continuous adjoint method," Computers \& Fluids 126, 12-24 (2016). 
[13] M Samimy, KBMQ Zaman, and MF Reeder, "Effect of tabs on the flow and noise field of an axisymmetric jet," AIAA journal 31, 609-619 (1993).

[14] William Thomson, "Ii. on vortex atoms," Lond. Edinb. Dubl. Phil. Mag. 34, 15-24 (1867).

[15] Sheila E Widnall and Chon-Yin Tsai, "The instability of the thin vortex ring of constant vorticity," Philos. Trans. R. Soc. A-Math. Phys. Eng. Sci. 287, 273-305 (1977).

[16] Sheila E Widnall, Donald B Bliss, and Chon-Yin Tsai, "The instability of short waves on a vortex ring," J. Fluid Mech. 66, 35-47 (1974).

[17] DW Moore and PG Saffman, "The instability of a straight vortex filament in a strain field," in Proc. R. Soc. A-Math. Phys. Eng. Sci., Vol. 346 (The Royal Society, 1975) pp. 413-425.

[18] Chon-Yin Tsai and Sheila E Widnall, "The stability of short waves on a straight vortex filament in a weak externally imposed strain field," J. Fluid Mech. 73, 721-733 (1976).

[19] JDA Walker, CR Smith, AW Cerra, and TL Doligalski, "The impact of a vortex ring on a wall," J. Fluid Mech. 181, 99-140 (1987).

[20] TT Lim and TB Nickels, "Instability and reconnection in the head-on collision of two vortex rings," Nature 357, 225-227 (1992).

[21] Paolo Orlandi and Roberto Verzicco, "Vortex rings impinging on walls: axisymmetric and three-dimensional simulations," J. Fluid Mech. 256, 615-646 (1993).

[22] Ming Cheng, Jing Lou, and Li-Shi Luo, "Numerical study of a vortex ring impacting a flat wall," J. Fluid Mech. 660, 430-455 (2010).

[23] Heng Ren and Xi-Yun Lu, "Dynamics and instability of a vortex ring impinging on a wall," Commun. Comput. Phys. 18, 1122-1146 (2015).

[24] Philippe Chatelain, Demosthenes Kivotides, and Anthony Leonard, "Reconnection of colliding vortex rings," Physical review letters 90, 054501 (2003).

[25] PG Saffman, "The number of waves on unstable vortex rings," J. Fluid Mech. 84, 635-639 (1978).

[26] Jian Deng, Lubao Teng, CP Caulfield, and Xuerui Mao, "Instabilities of interacting vortex rings generated by an oscillating disk," Phys. Rev. E 94, 033107 (2016).

[27] George Karniadakis and Spencer Sherwin, Spectral/hp element methods for computational fluid dynamics (Oxford University Press, 2013).

[28] Ramesh Natarajan and Andreas Acrivos, "The instability of the steady flow past spheres and disks," J. Fluid Mech. 254, 323-344 (1993).

[29] GJ Sheard, MC Thompson, and Kerry Hourigan, "From spheres to circular cylinders: the stability and flow structures of bluff ring wakes," J. Fluid Mech. 492, 147-180 (2003).

[30] G Rocco, TA Zaki, X Mao, H Blackburn, and SJ Sherwin, "Floquet and transient growth stability analysis of a flow through a compressor passage," Aerosp. Sci. Technol. (2015). 